\title{
Apsidal Precession Effects on the Lunar-Based Synthetic Aperture Radar Imaging Performance
}

\author{
Zhen $\mathrm{Xu}^{(}{ }^{\circledR}$, Student Member, IEEE, Kun-Shan Chen ${ }^{\circledR}$, Fellow, IEEE, \\ Zhao-Liang Li ${ }^{\circledR}$, Senior Member, IEEE, and Gen-Yuan Du
}

\begin{abstract}
There have been considerable interests in the lunar-based synthetic aperture radar (LBSAR) for monitoring large-scale geoscience phenomena. However, the signal distortions given rise by lunar orbital perturbations, especially the apsidal precession, are particularly severe in the LBSAR. The apsidal precession effects can induce a coordinate drift of the LBSAR, which can further lead to the variation in the range history of the LBSAR. As a result, LBSAR's image performance might be affected. In this letter, we thoroughly investigate whether the apsidal precession effects cause the phase decorrelation in the signal of the LBSAR, and how such effects impact the LBSAR imaging. The theoretical result shows that the impact of the lunar apsidal precession mainly results in the first-order and second-order Doppler errors, which further influence the geometric location and focusing quality along the azimuth direction. Numerical simulations using the point target response show good consistency with the theoretical analysis. To this end, the lunar apsidal precession effects deserve special care in the LBSAR for high imaging quality.
\end{abstract}

Index Terms-Apsidal precession, Doppler error, focusing quality, geometric location, lunar-based (LB) synthetic aperture radar (SAR), signal distortions.

\section{INTRODUCTION}

$\mathbf{I}$ $\mathrm{N}$ RECENT years, using the moon as a remote sensing platform has attracted growing attention due to its unique advantages for the earth observation [1]-[3]. As Earth's natural satellite, the moon can be regarded as a stable platform with a long life cycle. Accordingly, the lunar-based (LB) platform would be a fascinating supplement to the existing remote sensing systems, which can offer long-term continuous data for the earth observation [2]. Since the synthetic aperture radar (SAR) is capable of observing the earth under all-time and almost all-weather conditions, thus, it is chosen as one of the primary sensors built on the LB platform [4], [5].

Manuscript received December 30, 2019; revised February 14, 2020; accepted April 30, 2020. This work was supported in part by the National Natural Science Foundation of China under Grant 41590853, Grant 41590852, and Grant 41531175. (Corresponding author: Kun-Shan Chen).

Zhen $\mathrm{Xu}$ and Kun-Shan Chen are with the Institute of Remote Sensing and Digital Earth, Chinese Academy of Sciences, Beijing 100101, China, and also with the College of Resources and Environment, University of Chinese Academy of Sciences, Beijing 100049, China (e-mail: xuzhen@radi.ac.cn; chenks@radi.ac.cn).

Zhao-Liang $\mathrm{Li}$ is with ICube (UMR 7357) CNRS, UdS, CS 10413, 67412 Illkirch, France, and also with the Institute of Agricultural Resources and Regional Planning, Chinese Academy of Agricultural Sciences, Beijing 100081, China (e-mail:lizl@unistra.fr).

Gen-Yuan Du is with the School of Information Engineering, Xuchang University, Xuchang 461000, China.

Color versions of one or more of the figures in this letter are available online at http://ieeexplore.ieee.org.

Digital Object Identifier 10.1109/LGRS.2020.2992508
Today, scientific issues related to LB SAR (LBSAR) boast of an extensive literature [1], [4]-[10]. However, these works are mainly concentrated on the system configuration, signal modeling, and potential applications [8]. There is little literature concerning the orbital perturbation effects on the LBSAR imaging, although the LBSAR is sensitive to them [9]. It is acknowledged that the lunar orbit is influenced by many perturbed factors, e.g., precession, libration, and so on [11]. All these perturbation effects would result in variations of the idealized Kepler orbit; as a result, the motion of the SAR system relative to the target of interest is modified. The lunar apsidal precession, in particular, exerts a direct effect on the relative motion between the LBSAR and the ground target.

The precessional motion is the variation in the orientation of a rotational axis with respect to a reference plane [12]. The lunar orbit experiences two typical types of precession: the apsidal precession with a cycle of 8.85 years and the nodal precession with a period of 18.6 years [13]. The apsidal precession is the eastward precession of the lunar orbit's major axis, which would bring about temporal variation in the argument of perigee (AOP), and further causes a coordinate drift of LBSAR. Consequently, there are Doppler errors in the signal of the LBSAR. Consequently, a question arises whether or not the apsidal precession would present effects on the LBSAR imaging.

This letter aims to investigate whether or not the phase coherence of the LBSAR's signal is affected by the apsidal precession effects, and if so, how such effects impact the LBSAR imaging. In Section II, a description of the lunar apsidal precession is presented, based on which the Doppler errors due to the apsidal precession effects are derived. Section III provides the theoretical analysis of image distortions given rise by the apsidal precession effects. Following, in Section IV, the point target responses are simulated and evaluated for confirming the theoretical analysis. Finally, a conclusion is drawn in Section V.

\section{DOPPlER ERrors DUE TO THE APSIDAL PRECESSION EFFECTS}

Since the LBSAR is a remote sensing system installed on the lunar surface, thus, it revolves around the earth along its particular orbit. Here, the numerical lunar ephemeris from Development Ephemeris (DE) produced by the Jet Propulsion Laboratory (JPL) is used to provide orbital parameters of the lunar orbit; meanwhile, the Barycentric Dynamical Time is used to offer a coordinate time scale [14]. Note that there is a series of DE; the DE 430 is adopted in this letter.

Fig. 1 shows the AOP of the lunar orbit during one cycle of the lunar apsidal precession, from which we observe that the AOP is time-varying as a result of the apsidal precession effects during the long period. What interests us most is 


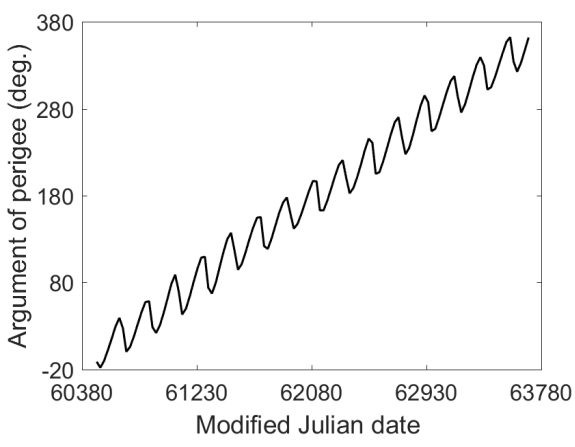

Fig. 1. Temporal variation in the AOP of the lunar orbit within one cycle of the lunar apsidal precession.

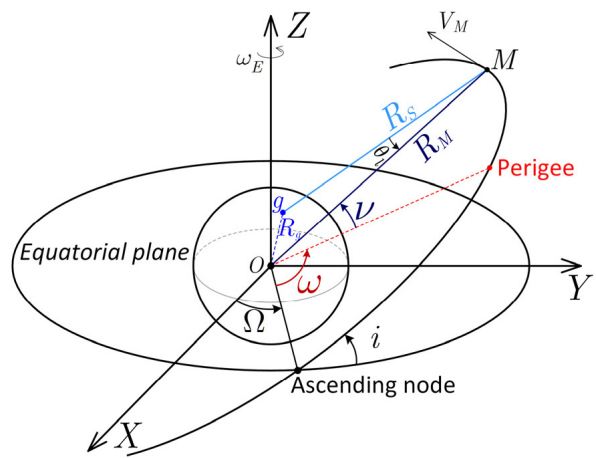

Fig. 2. Schematic of the LBSAR's imaging geometry.

TABLE I

PARAMETERS IN THE LBSAR'S IMAGING GEOMETRY

\begin{tabular}{c|c|c|c}
\hline Symbol & Parameters & Symbol & Parameters \\
\hline$M$ & Site of the LBSAR & $g$ & Target of interest \\
\hline$R_{g}$ & Earth's radius at $g$ & $R_{S}$ & Slant range \\
\hline$i$ & Orbital inclination & $\theta_{l}$ & SAR look angle \\
\hline$\omega$ & Argument of perigee (AOP) & $\nu$ & True anomaly \\
\hline$\Omega$ & $\begin{array}{c}\text { Right ascension of the } \\
\text { ascending node (RAAN) }\end{array}$ & $R_{M}$ & Earth-LBSAR Distance \\
\hline
\end{tabular}

that whether or not the short-term apsidal precession effects impact the phase coherence of the LBSAR's signal during the synthetic aperture time. To detail such effects, we analyze the range error due to the short-term apsidal precession based on the imaging geometry of the LBSAR shown in Fig. 2. The parameters appearing in the LBSAR's imaging geometry are summarized in Table I.

For clarity, the orbit parameters with and without the apsidal precession are assumed to be the same at the zero azimuth time (see Fig. 3). Hence, the coordinate drift given rise by the apsidal precession is presented in the following equation:

$$
\mathbf{R}_{\mathrm{drift}, \eta}=\mathbf{R}_{M, \eta}-\mathbf{R}_{M A, \eta}
$$

where $\mathbf{R}_{M, \eta}=\left[X_{M, \eta}, Y_{M, \eta}, Z_{M, \eta}\right]^{T}$ is the LBSAR's position vector at the azimuth time $\eta$. Note that the LBSAR's site is still subject to select at present [6], we may assume that the position of the LBSAR is the same as that of the moon for proceeding with this analysis. $\mathbf{R}_{M A, \eta}$, the position vector of the LBSAR without apsidal precession, takes the form of

$$
\begin{aligned}
\mathbf{R}_{\mathrm{MA}, \eta}= & \left|\mathbf{R}_{M, \eta}\right| \\
& \times\left[\begin{array}{c}
\cos \Omega_{\eta} \cos \left(\omega_{0}+v_{\eta}\right)-\sin \Omega_{\eta} \cos i_{\eta} \sin \left(\omega_{0}+v_{\eta}\right) \\
\sin \Omega_{\eta} \cos \left(\omega_{0}+v_{\eta}\right)+\cos \Omega_{\eta} \cos i_{\eta} \sin \left(\omega_{0}+v_{\eta}\right) \\
\sin i_{\eta} \sin \left(\omega_{0}+v_{\eta}\right)
\end{array}\right]
\end{aligned}
$$

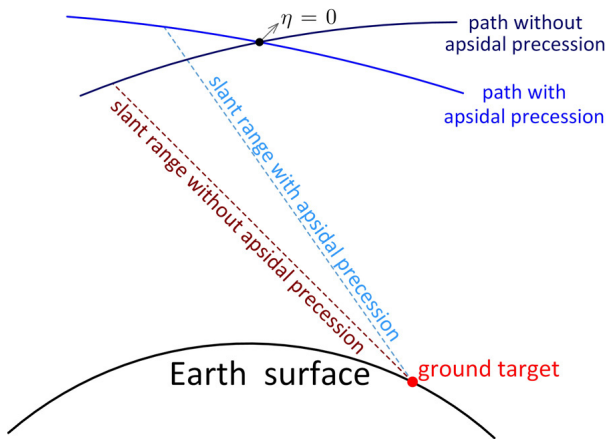

Fig. 3. Schematic of the LBSAR's range history with and without the lunar apsidal precession.

with

$$
\left\{\begin{array}{l}
i_{\eta}=\cos ^{-1}\left(W_{z, \eta} /\left|\mathbf{W}_{\eta}\right|\right) \\
\Omega_{\eta}=\tan ^{-1}\left(-W_{x, \eta} / W_{y, \eta}\right) \\
v_{\eta}=\cos ^{-1}\left(\frac{\left|\mathbf{W}_{\eta}\right|^{2}-\mu\left|\mathbf{R}_{M, \eta}\right|}{\sqrt{\mu^{2}\left|\mathbf{R}_{M, \eta}\right|^{2}-2 \mu\left|\mathbf{R}_{M, \eta}\right|\left|\mathbf{W}_{\eta}\right|^{2}+\left|\mathbf{R}_{M, \eta}\right|^{2}\left|\mathbf{V}_{M, \eta}\right|^{2}\left|\mathbf{W}_{\eta}\right|^{2}}}\right)
\end{array}\right.
$$

where $\mu$ is the gravitational coefficient. $W_{x, \eta}, W_{y, \eta}$, and $W_{z, \eta}$ are the $x, y$, and $z$ components of the areal velocity vector $\mathbf{W}_{\eta}$ at the azimuth time $\eta$, respectively, which take the following form [15]:

$$
\mathbf{W}_{\eta}=\mathbf{R}_{M, \eta} \times \mathbf{V}_{M, \eta}
$$

where $\mathbf{V}_{M, \eta}$ is the moon's velocity vector at the azimuth time $\eta$, and it can be also obtained from the JPL DE430.

In (2), $\omega_{0}$ is the AOP at the zero azimuth time, which is given by

$$
\omega_{0}=\sin ^{-1}\left[Z_{E, \eta=0} /\left(\left|\mathbf{R}_{M, \eta=0}\right| \cdot \sin i_{\eta=0}\right)\right]-v_{\eta=0}
$$

where $\mathbf{R}_{M, \eta=0}, Z_{M, \eta=0}, i_{\eta=0}, v_{\eta=0}$ are the orbital parameters at the zero azimuth time.

A range error is induced because of the coordinate drift, and it can be further expressed as

$$
\Delta R_{a p}(\eta)=\frac{2 \mathbf{R}_{\mathrm{drift}, \eta} \cdot\left(\mathbf{R}_{M 0, \eta}-\mathbf{R}_{g, \eta}\right)+\left|\mathbf{R}_{\mathrm{drift}, \eta}\right|^{2}}{\left|\mathbf{R}_{M 0, \eta}-\mathbf{R}_{g, \eta}\right|+\left|\mathbf{R}_{M, \eta}-\mathbf{R}_{g, \eta}\right|}
$$

where $\mathbf{R}_{g, \eta}$ is the ground target's position vector. It is preferred to determine the position of the ground target through the steering method for the LBSAR proposed in [10] to readjust the Doppler centroid to zero and, subsequently, to avoid pixel skewing that poses a challenge to geometric calibration [8].

To deal with the effects of lunar apsidal precession, the range error could be expressed in series form as

$$
\Delta R_{a p}(\eta)=\Delta R_{0}+\Delta R_{1} \eta+\Delta R_{2} \eta^{2}+\Delta R_{3} \eta^{3}+\cdots
$$

where $\Delta R_{0}$ is the constant part of the range error being equal to 0 since the orbit parameters with and without apsidal precession are same at the zero azimuth time (see Fig. 3). $\Delta R_{i}$ is the $i$ th-order $(i=1,2,3)$ Taylor series coefficient of the range error against the azimuth time given by

$$
\Delta R_{1}=-\lambda \Delta f_{d 1} / 2, \quad \Delta R_{2}=-\lambda \Delta f_{d 2} / 4, \quad \Delta R_{3}=-\lambda \Delta f_{d 3} / 12
$$

where $\lambda$ is the wavelength. $\Delta f_{d i}$, the $i$ th-order Doppler error due to the effects of the lunar apsidal precession, is given by

$$
\Delta f_{d i}=-\left.\frac{2}{\lambda} \frac{\partial^{i}\left[\Delta R_{a p}(\eta)\right]}{\partial \eta^{i}}\right|_{\eta=0} .
$$


TABLE II

SimUlation PARAMETERS FOR THE APSIDAL PRECESSION EFFECTS

\begin{tabular}{c|c|c|c}
\hline Symbol & Parameters & Quantity & Unit \\
\hline$R_{g}$ & Earth's radius & 6371.0 & $\mathrm{~km}$ \\
\hline$\omega_{E}$ & Earth's rotation angular velocity & $7.292 \times 10^{-5}$ & $\mathrm{rad} / \mathrm{s}$ \\
\hline$T_{s a r}$ & Synthetic aperture time & $50,150,300$ & $\mathrm{~s}$ \\
\hline$f_{c}$ & Carrier frequency & 1.2 & $\mathrm{GHz}$ \\
\hline$f_{d c}$ & $\begin{array}{c}\text { Doppler centroid with } \\
\text { apsidal precession }\end{array}$ & 0 & $\mathrm{~Hz}$ \\
\hline
\end{tabular}

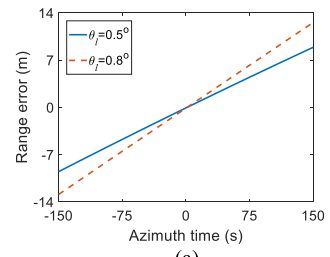

(a)

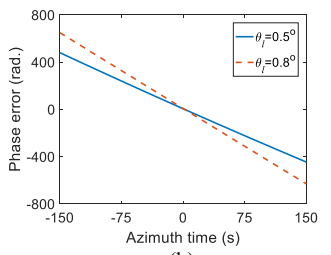

(b)
Fig. 4. Under the apsidal precession effects. (a) Range error against the azimuth time. (b) Phase error against the azimuth time.

The range and Doppler errors of the LBSAR under the apsidal precession effects are examined and illustrated below. The simulation parameters are given in Table II. For clarity but without loss of generality, the LBSAR is assumed to be looking from the left-hand side and the zero azimuth time is set to 00:00:00 on March 20, 2024, in the time coordinate. It is worth mentioning that when the Doppler steering method, i.e., [10], is applied, the image scene is pertinent to the SAR look angle, which stands at between $0.09^{\circ}$ and $0.91^{\circ}$ at a given zero azimuth time according to the bounds of the SAR look angle in [10]. Hence, the range of the SAR look angle is set to $\left[0.1^{\circ}, 0.9^{\circ}\right]$.

Fig. 4 shows the range error and corresponding phase error with various look angles $\left(0.5^{\circ}, 0.8^{\circ}\right)$ against the azimuth time, from which we observe that the range error increases with the increasing synthetic aperture time and look angle. Since the range error is time-varying, it turns out that the range history of the LBSAR is affected by the apsidal precession effects. Furthermore, the phase error is significantly large to deteriorate the LBSAR imaging performance.

Typically, the apsidal precession effects could manifest as the impacts of Doppler errors on the phase coherence of the LBSAR's signal. For a detailed analysis, Doppler errors from first-order to fourth-order against the SAR look angle are calculated and shown in Fig. 5.

It can be identified from Fig. 5 that the apsidal precession effects mainly induce the first-order and second-order Doppler errors, which could affect the LBSAR imaging if not properly compensated. On the contrary, the third-order and fourth-order Doppler errors are negligibly small, suggesting that neither of them impact the LBSAR imaging. Moreover, the size of the first-order Doppler error increases with the increasing SAR look angle; on the contrary, the scale of the second-order Doppler error decreases with the increasing look angle. Thus, it can be concluded that the relationship between the image shift and look angle is totally different from the relationship of the degree of image defocusing to the look angle, as will be elaborated in Section III.

Presently, we have analyzed the Doppler errors due to the apsidal precession effects. It is shown that the apsidal precession can mainly induce the first-order and second-order Doppler errors, which may impact the phase coherence and further affect the azimuth imaging of the LBSAR.

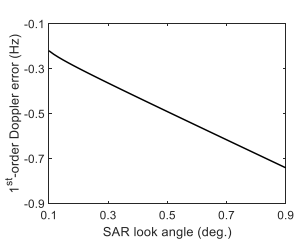

(a)

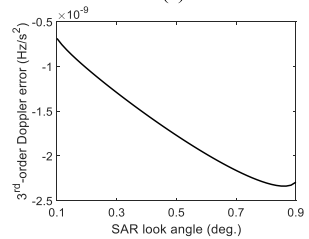

(c)
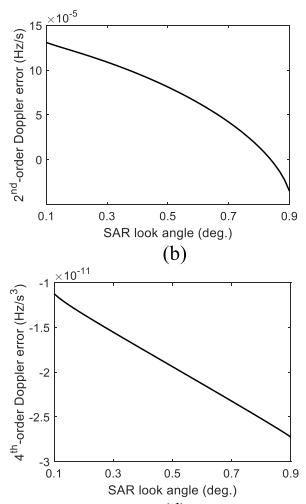

(d)
Fig. 5. Under the apsidal precession effects. (a) First-order Doppler error, (b) second-order Doppler error, (c) third-order Doppler error, and (d) forthorder Doppler error, with respect to the look angle of the LBSAR.

In Section III, the image distortions under the apsidal precession effects are theoretically analyzed.

\section{Theoretical Analysis of Image Distortion Due TO THE LUNAR APSIDAL PRECESSION}

From the SAR focusing theory, the first-order Doppler error could influence the ground target's geometric location along the azimuth direction. By comparison, the secondorder Doppler error would cause azimuth defocusing through raising the sidelobe as well as broadening the main lobe. Furthermore, the third-order Doppler error would bring about the asymmetric of the sidelobe, if it is potential enough.

\section{A. Azimuth Shift Due to the Lunar Apsidal Precession}

The first-order Doppler error could lead to an image shift along the azimuth direction; this image shift can be calculated as

$$
\Delta L_{a}=V_{g} \cdot \Delta f_{d 1} / f_{d 2}
$$

where $f_{d 2}$ denotes the Doppler frequency modulation (FM) rate of the LBSAR, and $V_{g}$ represents the ground velocity, which is defined as

$$
V_{g}=\left|\omega_{\mathbf{E}} \times \mathbf{R}_{g, \eta=0}-\right| \mathbf{R}_{g, \eta=0}\left|\frac{\left\langle\mathbf{R}_{M, \eta=0}, \mathbf{R}_{g, \eta=0}\right\rangle}{\left|\mathbf{R}_{M, \eta=0}\right| \cdot\left|\mathbf{R}_{g, \eta=0}\right|} \cdot \frac{\mathbf{V}_{M, \eta=0}}{\left|\mathbf{R}_{M, \eta=0}\right|}\right|
$$

where $\omega_{\mathbf{E}}=\left[0,0, \omega_{E}\right]^{T}$ and $\omega_{E}$ is the angular velocity of the earth's self-rotation. $\mathbf{R}_{g, \eta=0}, \mathbf{R}_{M, \eta=0}$, and $\mathbf{V}_{M, \eta=0}$ are, respectively, the target's position vector, LBSAR's position vector, and LBSAR's velocity vector at the zero azimuth time. The symbols " $\langle$,$\rangle " and " x$ " indicate the inner and cross products, respectively.

The azimuth shift versus the SAR look angle is shown in Fig. 6. As is seen, there is nonlinear dependence of the azimuth shift on the look angle. This phenomenon attributes that the first-order Doppler error increases, whereas the Doppler FM rate decreases with the increasing SAR look angle. In addition, the azimuth shift can easily be up from hundreds of meters to thousands of meters with the incremental increase of the SAR look angle. When the SAR look angle goes beyond $0.8^{\circ}$, a steep rise of the azimuth shift is observed and is hardly acceptable. 


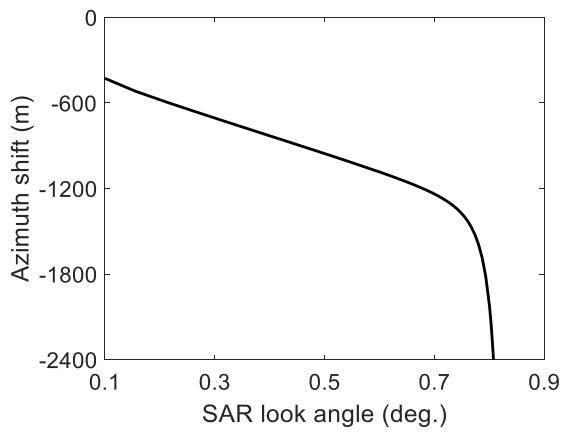

Fig. 6. Under the apsidal precession effects, the azimuth shift versus the SAR look angle.

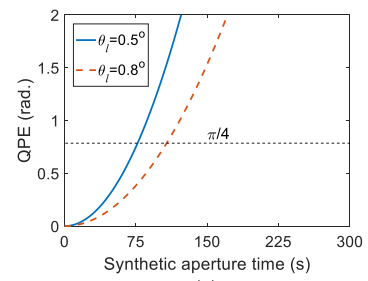

(a)

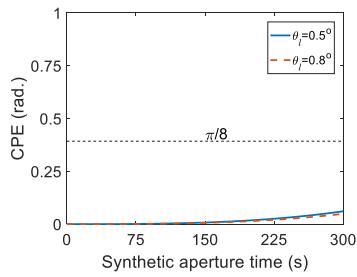

(b)
Fig. 7. Under the apsidal precession effects. (a) The relation between synthetic aperture time and QPE term. (b) The relation between synthetic aperture time and $\mathrm{CPE}$.

\section{B. Azimuth Defocusing Due to the Lunar Apsidal Precession}

The second-order Doppler error may cause defocusing and resolution degradation along the azimuth direction in the LBSAR. Here, we define the quadratic phase error (QPE) to measure this effect

$$
\mathrm{QPE}=\pi \cdot\left|\Delta f_{d 2}\right| \cdot T_{\mathrm{sar}}^{2} / 4
$$

where $T_{\text {sar }}$ is the synthetic aperture time of the LBSAR.

For a more accurate analysis, we also take the third-order Doppler error into account. The cubical phase error (CPE) due to the third-order Doppler error is written as

$$
\mathrm{CPE}=\pi \cdot\left|\Delta f_{d 3}\right| \cdot T_{\text {sar }}^{3} / 24 .
$$

As a rule of thumb, the threshold values for the QPE and CPE are $\pi / 4$ and $\pi / 8$, respectively.

The QPE and CPE may cause a defocusing phenomenon along the azimuth direction; however, both of them depend on the synthetic aperture time of the LBSAR. To make an exhaustive analysis, the relationship between the QPE and CPE and the synthetic aperture time is shown in Fig. 7.

It can be seen in Fig. 7 that both the QPE and CPE are positively correlated with the SAR look angle. In addition, the CPE rarely exceeds the threshold of $\pi / 8$, implying that there is little impact of the CPE on the LBSAR imaging. On the contrary, the effect of the QPE is indeed not negligible; under this effect, the focusing performance highly depends on the exposure time of the LBSAR. The QPE is always smaller than its threshold value when the exposure time is shorter than $75 \mathrm{~s}$, suggesting that the LBSAR imaging is little disturbed by the QPE within a short synthetic aperture time. But for the LBSAR with a synthetic aperture time longer than $75 \mathrm{~s}$, the QPE must be taken into account in the signal processing of the LBSAR.

\section{Numerical Simulations}

For validating the theoretical analysis, the point target responses under the apsidal precession effects are simulated
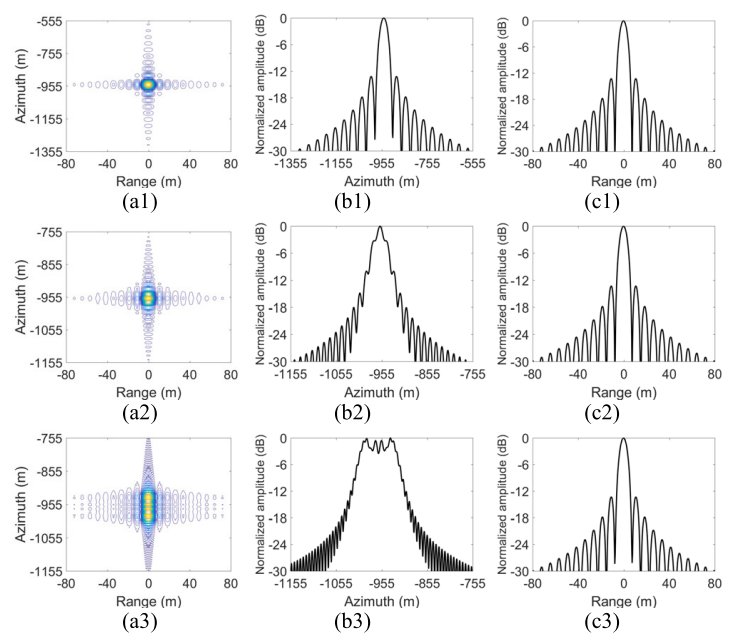

Fig. 8. Under the apsidal precession effects, the point target response of the LBSAR with a look angle of $0.5^{\circ}$, where the first, second, and third rows show an exposure time of 50,150, and $300 \mathrm{~s}$, respectively. (a1) Contour. (b1) Azimuth profile. (c1) Range profile. (a2) Contour. (b2) Azimuth profile. (c2) Range profile. (a3) Contour. (b3) Azimuth profile. (c3) Range profile.

\section{TABLE III}

Quality Measure of a Point Target Response

\begin{tabular}{|c|c|c|c|c|}
\hline Direction & \begin{tabular}{|ll} 
Parameters & $T_{\text {sar }}$ \\
\end{tabular} & $50 \mathrm{~s}$ & $150 \mathrm{~s}$ & $300 \mathrm{~s}$ \\
\hline \multirow{5}{*}{ Azimuth } & Ideal resolution $(\mathrm{m})$ & 38.95 & 12.98 & 6.49 \\
\hline & Real resolution $(\mathrm{m})$ & 38.97 & 20.16 & - \\
\hline & Image shift (m) & -955.37 & -955.37 & - \\
\hline & PSLR (dB) & -13.16 & -3.24 & - \\
\hline & $\operatorname{ISLR}(\mathrm{dB})$ & -13.15 & -3.20 & - \\
\hline \multirow{5}{*}{ Range } & Ideal resolution $(\mathrm{m})$ & 7.50 & 7.50 & 7.50 \\
\hline & Real resolution $(\mathrm{m})$ & 7.49 & 7.49 & 7.49 \\
\hline & Image shift (m) & 0.00 & 0.00 & 0.00 \\
\hline & PSLR (dB) & -13.24 & -13.25 & -13.28 \\
\hline & ISLR (dB) & -13.15 & -13.14 & -13.17 \\
\hline
\end{tabular}
With A LOOK ANGLE OF $0.5^{\circ}$

and evaluated in this section. To begin with, Fig. 8 shows the point target responses under different exposure times $(50,150$, and $300 \mathrm{~s}$ ). The SAR look angle is set to $0.5^{\circ}$ and the original position of the target is at $(0,0 \mathrm{~m})$. The quality measures are summarized in Table III.

From Fig. 8 and Table III, the apsidal precession bears a negligible impact on the range imaging. On the contrary, the effects of the apsidal precession on the azimuth imaging are quite significant. The point target displaces from the scene center due to the azimuth shift. In addition, the apsidal precession effects do not defocus the azimuth imaging when the exposure time is short, e.g., 50 s. For LBSAR with a longer synthetic aperture time, e.g., $150 \mathrm{~s}$, both the azimuth peak to sidelobe ratio (PSLR) and integrated sidelobe ratio (ISLR) rise to -3.24 and $-3.20 \mathrm{~dB}$, respectively. Meanwhile, the azimuth resolution is severely degraded due to the apsidal precession. When the exposure time of the LBSAR continues to increase, the defocusing becomes even more serious. Consequently, it is challenging to produce a high-quality image in the LBSAR due to the apsidal precession effects, particularly for a longer exposure time.

For a more rigorous analysis, using the same sets of exposure time, the point target responses of the LBSAR with a look angle of $0.8^{\circ}$ are simulated and shown in Fig. 9. The position of $(0 \mathrm{~m}, 0 \mathrm{~m})$ is used as a reference for the point 


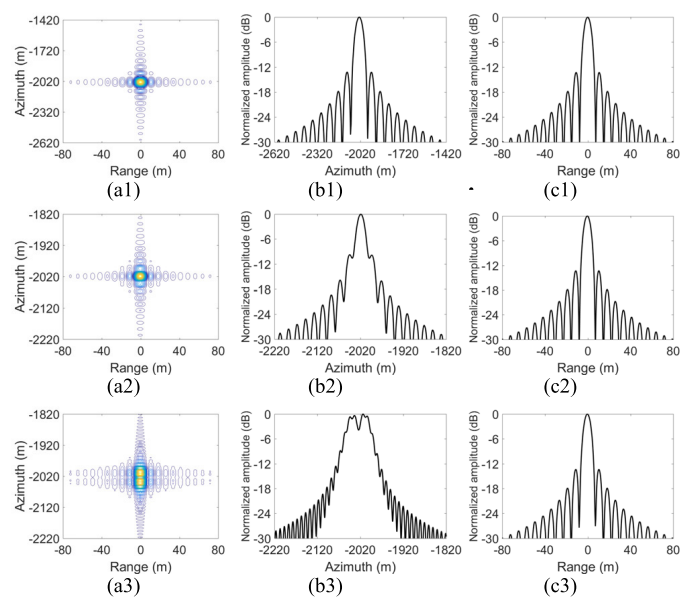

Fig. 9. Under the apsidal precession effects, the point target response of the LBSAR with a look angle of $0.8^{\circ}$, where the first, second, and third rows show an exposure time of 50, 150, and $300 \mathrm{~s}$, respectively. (a1) Contour. (b1) Azimuth profile. (c1) Range profile. (a2) Contour. (b2) Azimuth profile. (c2) Range profile. (a3) Contour. (b3) Azimuth profile. (c3) Range profile.

TABLE IV

Quality Measure of a Point Target Response WITH A LOOK ANGLE OF $0.8^{\circ}$

\begin{tabular}{|c|c|c|c|c|}
\hline Direction & \begin{tabular}{|ll} 
Parameters & $T_{\text {sar }}$ \\
\end{tabular} & $50 \mathrm{~s}$ & $150 \mathrm{~s}$ & $300 \mathrm{~s}$ \\
\hline \multirow{5}{*}{ Azimuth } & Ideal resolution $(\mathrm{m})$ & 59.63 & 19.88 & 9.94 \\
\hline & Real resolution $(\mathrm{m})$ & 59.72 & 21.15 & - \\
\hline & Image shift (m) & -2021.61 & -2021.61 & - \\
\hline & PSLR (dB) & -13.20 & -9.73 & - \\
\hline & $\operatorname{ISLR}(\mathrm{dB})$ & -13.38 & -9.32 & - \\
\hline \multirow{5}{*}{ Range } & Ideal resolution $(\mathrm{m})$ & 7.50 & 7.50 & 7.50 \\
\hline & Real resolution $(\mathrm{m})$ & 7.52 & 7.49 & 7.49 \\
\hline & Image shift (m) & 0.00 & 0.00 & 0.00 \\
\hline & PSLR (dB) & -13.24 & -13.25 & -13.32 \\
\hline & $\operatorname{ISLR}(\mathrm{dB})$ & -14.45 & -13.18 & -13.15 \\
\hline
\end{tabular}

target's original position in this case. The quality measures of simulation results are listed in Table IV.

As can be seen in Fig. 9 and Table IV, the effects of the apsidal precession remain profound for the LBSAR with a larger look angle. It can be recognized that the scale of the azimuth shift with a look angle of $0.8^{\circ}$ can be up to thousands of meters, which is more than enough to displace the target along the azimuth direction. In addition, a comparison of Figs. 8 and 9 shows that the scale of the azimuth shift is positively correlated with the SAR look angle. Interestingly, there is a negative correlation between the degree of the azimuth defocusing and the SAR look angle.

At this point, image distortions due to the apsidal precession effects are numerically analyzed; all results presented so far confirm the theoretical analysis. It is evident that the apsidal precession effects cannot be ignored in line with the focusing quality for an exposure time longer than $75 \mathrm{~s}$. The exposure time of $50 \mathrm{~s}$ could achieve azimuth resolutions of the LBSAR from 40 to $60 \mathrm{~m}$. On the other hand, the image quality is barely affected by the apsidal precession effects under such an exposure time. Since the large-scale phenomena arouse more attention in the earth observation from the LB platform [4]-[6], as a tradeoff, an exposure time of $50 \mathrm{~s}$ perhaps is an excellent choice for good image quality. If the geometric fidelity is intolerable for specific applications, it is better to compensate for the positioning error induced by the lunar apsidal precession effects through an accurate orbital measurement.

\section{CONClusion}

In this letter, the apsidal precession effects on the LBSAR imaging are investigated. The theoretical analysis shows that the range imaging is little disturbed by the apsidal precession effects. On the contrary, the azimuth imaging is profoundly affected due to the first-order and second-order Doppler errors given rise by the apsidal precession effects. In particular, the azimuth shift resulted from the first-order Doppler error ranges from hundreds of meters to thousands of meters, which is not acceptable for most applications. Furthermore, the azimuth focusing quality is obviously deteriorated by the second-order Doppler error when the exposure time is longer than $75 \mathrm{~s}$. The numerical simulations of the point target responses show good consistency with the theoretical analysis. Since the focusing quality under the apsidal precession effects becomes intolerable, an exposure time of $50 \mathrm{~s}$ is preferred in the design of LBSAR for an excellent image quality. For improving the geometric location, it is desirable to employ an accurate orbit measurement. Since the eccentricity perturbation of the lunar orbit might defocus the LBSAR imaging, it will be the priority in our future study.

\section{REFERENCES}

[1] M. Ramsey. (Mar. 2007). ESS Findings: Lunar ESS Findings: Lunar Science Planning and Workshop Overview. Accessed: Mar. 21, 2018. [Online]. Available: https://www.lpi.usra.edu/meetings/ LEA/presentations/closing_plenary/Ramsey_ESS_summary_20070302. pdf

[2] H. Ye, H. Guo, G. Liu, Q. Guo, and J. Huang, "Looking vector direction analysis for the moon-based Earth observation optical sensor," IEEE J. Sel. Topics Appl. Earth Observ. Remote Sens., vol. 11, no. 11, pp. 4488-4499, Nov. 2018.

[3] H. Ye, H. Guo, G. Liu, J. Ping, and Q. Guo, "Impacts of Platform's position errors on geolocation for a moon-based sensor," IEEE Geosci. Remote Sens. Lett., vol. 17, no. 1, pp. 112-116, Jan. 2020, doi: 10.1109/LGRS.2019.2916789.

[4] A. Moccia and A. Renga, "Synthetic aperture radar for Earth observation from a lunar base: Performance and potential applications," IEEE Trans. Aerosp. Electron. Syst., vol. 46, no. 3, pp. 1034-1051, Jul. 2010.

[5] G. Fornaro, G. Franceschetti, F. Lombardini, A. Mori, and M. Calamia, "Potentials and Limitations of Moon-Borne SAR Imaging," IEEE Trans. Geosci. Remote Sens., vol. 48, pp. 3009-3019, Apr. 2010.

[6] Z. Xu and K. S. Chen, "On signal modeling of moon-based SAR imaging of Earth," Remote Sens., vol. 10, no. 3, p. 486, Mar. 2018.

[7] Z. Xu and K.-S. Chen, "Temporal-spatial varying background ionospheric effects on the moon-based synthetic aperture radar imaging: A theoretical analysis," IEEE Access, vol. 6, pp. 66767-66786, 2018.

[8] Z. Xu and K.-S. Chen, "Effects of the Earth's curvature and lunar revolution on the imaging performance of the moon-based synthetic aperture radar," IEEE Trans. Geosci. Remote Sens., vol. 57, no. 8, pp. 5868-5882, Aug. 2019.

[9] Z. Xu, K.-S. Chen, and G. Zhou, "Effects of the Earth's irregular rotation on the moon-based synthetic aperture radar imaging," IEEE Access, vol. 7, pp. 155014-155027, 2019.

[10] Z. Xu, K.-S. Chen, and G. Zhou, "Zero-Doppler centroid steering for the moon-based synthetic aperture radar: A theoretical analysis," IEEE Geosci. Remote Sens. Lett., early access, Oct. 1, 2019, doi: 10.1109/LGRS.2019.2941505.

[11] M. C. Gutzwiller, "Moon-Earth-sun: The oldest three-body problem," Rev. Modern Phys., vol. 70, no. 2, pp. 589-639, Apr. 1998.

[12] R. R. Bate, D. D. Mueller, and J. E. White, Fundamentals of Astrodynamics. New York, NY, USA: Courier Corporation, 1971.

[13] I. D. Haigh, M. Eliot, and C. Pattiaratchi, "Global influences of the 18.61 year nodal cycle and 8.85 year cycle of lunar perigee on high tidal levels," J. Geophys. Res., vol. 116, no. C6, 2011, Art. no. C06025.

[14] W. M. Folkner, J. G. Williams, D. H. Boggs, R. S. Park, and P. Kuchynka, "The planetary and lunar ephemerides DE430 and DE431," Interplanetary Netw. Progr. Rep., vol. 196, pp. 1-81, Feb. 2014.

[15] O. Montenbruck and E. Gill, Satellite orbits: Models, Methods and Applications. Berlin, Germany: Springer, 2012. 